\title{
On Application Of Modified Gradient To Extended Conjugate Gradient Method Algorithm For Solving Optimal Control Problems
}

\author{
${ }^{1}$ K. J. ADEBAYO ${ }^{2}$ F. M. ADERIBIGBE and ${ }^{3}$ M. F. AKINMUYISE \\ ${ }^{1 \& 2}$ Department of Mathematical Sciences, Ekiti State University, Ado Ekiti, Nigeria. \\ ${ }^{3}$ Department of Mathematics, Adeyemi College of Education, Ondo, Ondo State, Nigeria.
}

\begin{abstract}
This paper investigates and discusses modification on the gradient of continuous function input introduced to Extended Conjugate Gradient Method algorithm (ECGM) employed in solving optimal control problems with the state variable constrained by a differential equation. Numerical results show some improvement over the classical methods.
\end{abstract}

Keywords: Optimal Control, Control Operator, Conjugate Gradient Method and Extended Conjugate Gradient Method.

\section{Introduction}

One of the commonly used technique for solving systems of linear equations is Gaussian elimination. It is referred to as a "direct" method because it determines the solution in a fixed number of arithmetic operations that can be predicted in advance. "Iterative" methods, on the other hand, do not have fixed costs since the solution is obtained from a sequence of approximate solutions, and the algorithm is terminated when some measure of the error has been made adequately small.

However, iterative methods are valuable tool for solving large systems of linear equations. They have several potential advantages over direct method. First, since the coefficient matrix need not be factored, there are no fill-in and loss of sparsely. Second, storage requirements are more often lower for iterative methods than for direct methods. In some cases, it may not be necessary to store the coefficient matrix at all. Third, if a good approximation to the coefficient matrix is available, and this approximation can be inverted at low cost, then an iterative method can take advantage of this information to obtain the solution more rapidly. This is not normally possible with a direct method. A great many iterative methods have been invented, but we will only consider one ofsuch: the ConjugateGradient Method (CGM). (Many of the other iterative methods are applied primarily in the solution of differential equations.) The ConjugateGradient Method is designed to solve

$$
A x=b
$$

In the case where the matrix $A$ is symmetric and positive definite. It can be considered as a technique for solving the equivalent problem

\section{Problem (P1)}

$$
\text { Minimize } f(x)=\frac{1}{2} x^{T} A x-b^{T} x
$$

The Conjugate Gradient Method (CGM) is a variant of the gradient method. In its simplest form, the gradient method uses the iterative scheme:

$$
x_{i+1}=x_{i}-\alpha \nabla f(x)
$$

to generate a sequence $\left\{x_{i}\right\}_{i=1}^{n}$ of vectors which converge to the minimum of $f(x)$. The parameter $\alpha$ appearing in (1.3) denotes the step length of the descent direction sequence. In particular, if $\mathrm{F}$ is a function on a Hilbert space $\mathcal{H}$ such that in $\mathcal{H}, \mathrm{F}$ admits a Taylor series expansion

$$
f(x)=f_{0}+\langle a, x\rangle_{\mathcal{H}}+\frac{1}{2}\langle x, Q x\rangle_{\mathcal{H}}
$$

where $a, x \in \mathcal{H}$ and is a positive definite, symmetric, linear operator, then it can be shown by [4] that $f$ possesses a unique minimum $x^{*}$ say in $\mathcal{H}$, and that

$\nabla f\left(x^{*}\right)=0$. The CGM algorithm for iteratively locating the minimum $x^{*}$ of $f(x)$ in $\mathcal{H}$ as described by [4] is as follows:

Step 1: Guess the first element $x_{0} \in \mathcal{H}$ and compute the remaining members of the sequence with the aid of the formulae in the steps 2 through 6.

Step 2: Compute the descent direction $p_{0}=-g_{0}$

Step 3: Set $x_{i+1}=x_{i}+\alpha_{i} p_{i}$; where $\alpha_{i}=\frac{\left\langle g_{i}, g_{i}\right\rangle_{\mathcal{H}}}{\left\langle p_{i}, Q p_{i}\right\rangle_{\mathcal{H}}}$

$1.5 \mathrm{~b}$

Step 4: Compute $g_{i+1}=g_{i}+\alpha_{i} Q p_{i}$ 
Step 5: $\operatorname{Set} p_{i+1}=-g_{i+1}+\beta_{i} p_{i} ; \beta_{i}=\frac{\left\langle g_{i+1}, g_{i+1}\right\rangle_{\mathcal{H}}}{\left\langle g_{i}, g_{i}\right\rangle_{\mathcal{H}}}$

Step 6: If $g_{i}=0$ for some $i$, then, terminate the sequence; else set $i=i+1$ and go to step 3 .

In the iterative steps 2 through 6 above, $p_{i}$ denotes the descent direction at $i$-th step of the algorithm, $\alpha_{i}$, is the step length of the descent sequence $\left\{x_{i}\right\}$ and $g_{i}$ denotes the gradient of $F$ at $x_{i}$. Steps 3, 4 and 5 of the algorithm reveal the crucial role of the linear operator $\mathrm{Q}$ in determining the step length of the descent sequence and also in generating a conjugate direction of search. Since the aim of this paper is investigating the general method for finding the gradient of a function, it is pertinent to discuss the modified gradient technique for the cases of a continuous function input as it applies to the CGM in the next section.

\section{Continuous Function Input Gradient}

We make the specific assumption that $\mathbb{U}$, the space in which the minimum of the cost functional is sought, is the space of continuous functions over the time interval $\left[t_{0}, t_{f}\right]$. We have then

$u=u(t), t \in\left[t_{0}, t_{f}\right]$

where $u(t)$ is continuous over $\left[t_{0}, t_{f}\right]$. We now seek the gradient of the cost functional in such a space of functions. Our approach is by

$$
\begin{aligned}
\left.\frac{d}{d x} J[u+\varepsilon z]\right|_{\varepsilon=0} & =\langle g(u)\rangle \\
& =\left\langle\nabla_{x} \emptyset\left(x\left(t_{f}, u\right)\right), \frac{d}{d x} x\left(t_{f}, u\right)\right\rangle
\end{aligned}
$$

which requires the evaluation of $\frac{d x}{d x}$.

Based on this, we consider

$\dot{x}=f(x, u): \quad x\left(t_{0}\right)=C$

where now $x \in \mathfrak{R}^{n}$ and $u \in \mathbb{U}$, a Hilbert space. $f(.,$.$) is a mapping from \mathfrak{R}^{n} \times \mathbb{U}$ to $\mathfrak{R}^{n} \cdot x\left(t_{0}\right)$, the state at the initial time, $t_{0}$, is assumed given as the fixed vector C. $x$ is called the state of the system and $u$, the control input. On integrating (2.3), we obtained

$x(t, u)=x\left(t_{0}\right)+\int_{t_{0}}^{t} f(x, u) d \tau$

We look inturn at the final state for an input $u+\varepsilon z$ where $z$ is thesame form as $u$ of (2.1), that is, $z=z(t), t \in\left[t_{0}, t_{f}\right]$. Thus again from (2.3)

$x\left(t_{f}, u+\varepsilon z\right)=x\left(t_{0}\right)+\int_{t_{0}}^{t} f(x, u+\varepsilon z) d \tau$

On differentiating (2.5) with respect to $\varepsilon$, we have

$\left.\frac{d}{d \varepsilon} x\left(t_{f}, u+\varepsilon z\right)\right|_{\varepsilon=0}=\left.\frac{d}{d x} x\left(t_{0}\right)\right|_{\varepsilon=0}+\left.\int_{t_{0}}^{t}\left\{f_{x}(x, u) \frac{d}{d x} x(t, u)+f_{u}(x, u) z(t)\right\} d t\right|_{\varepsilon=0}$

where $x(t, u)$ is as given by (2.4) and $f_{x}$ and $f_{u}$ are matrix and vector, respectively, whose elements are given by

$\left(f_{x}(x, u)\right)_{i, j}=\frac{\partial f_{i}}{\partial x}(x, u), \quad i, j=1,2, \ldots, n$

$\left(f_{u}(x, u)\right)_{i}=\frac{\partial f_{i}}{\partial u}(x, u), \quad i=1,2, \ldots, n$

The arguments $x$ and $u$, of $f$ have are functions of time given by (2.4) and (2.1) respectively. $f_{i}$ is the ith component of the vector value function $f$.

For a more tractable expression, we eliminate some of the arguments in (2.6), to get

$\frac{d}{d \varepsilon} x\left(t_{f}, u\right)=\frac{d}{d x} x\left(t_{0}\right)+\left.\int_{t_{0}}^{t_{f}}\left\{f_{x} \frac{d}{d x} x(t, u)+f_{u} z(t)\right\} d t\right|_{\varepsilon=0}$.

It can be seen that $\frac{d x}{d \varepsilon}$, which is needed to eliminate the gradient by (2.2), is the solution to the integral of (2.9) must hold for arbitrary $t_{f}>t_{0}$ since $t_{f}$ has been unspecified so far. Thus, by letting $t_{f}=t$ in (2.9) and differentiating with respect to it, we get the differential equation

$$
\begin{aligned}
& \frac{d}{d \varepsilon} x(t, u)=\frac{d}{d x} x\left(t_{0}\right)+\int_{t_{0}}^{t}\left\{f_{x} \frac{d}{d x} x(t, u)+f_{u} z(t)\right\} d t \\
& \frac{d}{d t} \frac{d}{d \varepsilon} x(t, u)=\frac{d}{d t} \frac{d}{d x} x\left(t_{0}\right)+\frac{d}{d t} \int_{t_{0}}^{t}\left\{f_{x} \frac{d}{d x} x(t, u)+f_{u} z(t)\right\} d t \\
& \frac{d}{d t} \frac{d}{d \varepsilon} x(t, u)=f_{x} \frac{d}{d x} x(t, u)+f_{u} z(t) \\
& \text { which } \frac{d x}{d \varepsilon} \text { must satisfy. }
\end{aligned}
$$

A standard linear, time varying differential equation of the form

$\dot{x}=C(t) x+D(t) u$

for which the solution is considered as

$x(t)=\emptyset\left(t, t_{0}\right) x\left(t_{0}\right)+\int_{t_{0}}^{t} \emptyset(t, \tau) B(\tau) u(\tau) d \tau$

And applying the solution (2.12) to (2.10) gives

$\frac{d}{d \varepsilon} x(t, u)=\varnothing\left(t, t_{0}\right) \frac{d}{d \varepsilon} x\left(t_{0}\right)+\int_{t_{0}}^{t} \emptyset(t, \tau) f_{u} z(\tau) d \tau$ 
where $\emptyset$ is the function of $t$ and $t_{0}$ that satisfies

$\frac{d}{d t} \emptyset\left(t, t_{0}\right)=f_{x} \emptyset\left(t, t_{0}\right): \emptyset\left(t_{0}, t_{0}\right)=I$

where $\mathrm{I}$ is the $n \times n$ identity matrix.

Then we substitute (2.13) into (2.2) to evaluate the desired gradient thus

$\left.\frac{d}{d x} J[u+\varepsilon z]\right|_{\varepsilon=0}=\left\langle\nabla_{x} \varnothing\left(x\left(t_{f}, u\right)\right), \emptyset\left(t, t_{0}\right) \frac{d}{d \varepsilon} x\left(t_{0}\right)+\int_{t_{0}}^{t} \emptyset(t, \tau) f_{u} z(\tau) d \tau\right\rangle$

Since $x\left(t_{0}\right)=K$, a constant, hence $\frac{d}{d \varepsilon} x\left(t_{0}\right)=0$ then,

$$
\begin{aligned}
\left.\frac{d}{d x} J[u+\varepsilon z]\right|_{\varepsilon=0} & =\langle g(u), z\rangle \\
& =\left\langle\nabla_{x} \emptyset\left(x\left(t_{f}, u\right)\right), \int_{t_{0}}^{t} \emptyset(t, \tau) f_{u} z(\tau) d \tau\right\rangle \\
& =\int_{t_{0}}^{t_{f}}\left\langle f_{u}^{T} \emptyset^{T}\left(t_{f}, \tau\right) \nabla_{x} \emptyset\left(x\left(t_{f}, u\right), z(\tau)\right\rangle d t\right.
\end{aligned}
$$

Let $\lambda(t)=\emptyset^{T}\left(t_{f}, \tau\right) \nabla_{x} \emptyset\left(x\left(t_{f}, u\right)\right.$

Putting (2.17) in (2.16), we obtain

$\langle g(u), z\rangle=\int_{t_{0}}^{t_{f}} f_{u}^{T} \lambda(\tau) z(\tau) d \tau$.

The integration of the right hand side of (2.18), which is the scalar product on the spaceof functions under consideration, shows the gradient of the cost functional for this case to be

$g(u(t))=f_{u}^{T} \lambda(t), \quad t \in\left[t_{0}, t_{f}\right]$

which is the desired result. Equation (2.19) is the gradient required to do CGM of the cost functional in the space of input control functions considered here.

Computation of the gradient as in (2.19) can be greatly simplified by recognizing that $\lambda(t)$ as given by (2.17) satisfies

$\dot{\lambda}(t)=-f_{x}^{T} \lambda(t): \quad \lambda\left(t_{f}\right)=\nabla_{x} \varnothing\left(x\left(t_{f}, u\right)\right.$

The system of differential equations in (2.20) is called the adjoint system to the system (2.3). Applicability of the CGM algorithm thus rests heavily on the explicit knowledge of the operator Q based on the introduction of the modified gradient technique to the CGM.

\section{The Extended Conjugate Gradient Method (ECGM)}

According to [2], an extended conjugate gradient method (ECGM) adopts the CGM to obtain the solution of a scalar, linear, optimal control problem of the form:

\section{Problem (P2)}

$\min _{(x, u)} \int_{0}^{T}\left\{A x^{2}(t)+B u^{2}(t)\right\} d t$

Subject to the constraint

$$
\begin{aligned}
& \dot{x}(t)=C x(t)+D u(t) ; 0 \leq t \leq T \\
& x(0)=x_{0}
\end{aligned}
$$

where $\mathrm{A}, \mathrm{B}, \mathrm{C}$ and $\mathrm{D}$ are specified constants such that $A>0, B>0 ; x_{0}$ and $\mathrm{T}$ are given, $\dot{x}(t)$ denotes the derivative of the state vector, $x($.$) , with respect to time and u($.$) is the control vector. As conventional with$ penalty function techniques, (3.1) and (3.2) may equivalently be written

$\min _{(x, u)} \int_{0}^{T}\left\{A x^{2}(t)+B u^{2}(t)+\mu\|\dot{x}(t)-C x(t)+D u(t)\|^{2}\right\} d t, \mu>0$

where $\mu$, is the penalty parameter and $\mu\|\dot{x}(t)-C x(t)+D u(t)\|^{2}$ is the penalty term. The operator, Q, was developed by [6] and was later improved upon by [1], which is related to the problem in the sense that:

$\langle y, Q y\rangle_{\mathcal{H}} \cong \min _{(x, u)} \int_{0}^{T}\left\{A x^{2}(t)+B u^{2}(t)+\mu\|\dot{x}(t)-C x(t)+D u(t)\|^{2}\right\} d t$,

where $\mathcal{H}$ is a suitably chosen Hilbert space. The operator $\mathrm{Q}$ is then utilized in the iterative procedure of the CGM in order to arrive at a solution of the problem (P2). Generally, according to [1], for discrete type optimization problems which satisfy the hypotheses on

$$
f(x)=f_{0}+\langle a, x\rangle_{\mathcal{H}}+\frac{1}{2}\langle x, Q x\rangle_{\mathcal{H}}
$$

The linear operator Q is readily determined (to see [4] pp. 51 - 53); and such problems enjoy the beauty of the CGM as a computational scheme since the CGM exhibits quadratic convergence and requires only a little more computation per iteration.

According to [6], the operator $Q$ is such that

$$
(\mathrm{Q} z)(t) \equiv\left[\begin{array}{ll}
Q_{11} & Q_{12} \\
Q_{21} & Q_{22}
\end{array}\right]\left[\begin{array}{l}
x(t) \\
u(t)
\end{array}\right]=\left[\begin{array}{l}
\left(Q_{11} x\right)(t)+\left(Q_{12} u\right)(t) \\
\left(Q_{21} x\right)(t)+\left(Q_{22} u\right)(t)
\end{array}\right]
$$

with the composite operators $Q_{11}, Q_{12} Q_{21}$ and $Q_{22}$ given by

$$
\begin{aligned}
\left(Q_{11} x\right)(t)= & -\mu[\dot{x}(0)-C x(0)] \operatorname{Sinh}(\mathrm{t})+\mu \int_{0}^{t}[\dot{x}(s)-C x(s)] \operatorname{Cosh}(t-s) d s \\
& -\int_{0}^{t}\left[\left(A+\mu C^{2}\right) x(s)-\mu C \dot{x}(s)\right] \operatorname{Sinh}(t-s) d s
\end{aligned}
$$




$$
\begin{aligned}
+ & {\left[\left(a+\mu C^{2}\right) x(0)-\mu C \dot{x}(0)\right] \operatorname{Cosh}(t)+\frac{\operatorname{Sinh}(t)}{\operatorname{Sinh}(T)}\left\{\left(a+\mu C^{2}\right) x(T)-\mu C \dot{x}(T)\right.} \\
& +\mu \operatorname{Sinh}(T)[\dot{x}(0)-C x(0)]-\mu \int_{0}^{T}[\dot{x}(s)-C x(s)] \operatorname{Cosh}(T-s) d s \\
& \left.+\int_{0}^{T}\left[\begin{array}{c}
\left(A+\mu C^{2}\right) x(s) \\
-\mu C \dot{x}(s)
\end{array}\right] \operatorname{Sinh}(T-s) d s-\left[\left(A+\mu C^{2}\right) x(0)-\mu C \dot{x}(0)\right] \operatorname{Cosh}(T)\right\}, 0 \leq t \leq T \quad 3.5 \\
\left(Q_{21} x\right)(t)= & \mu C D x(t)-\mu D \dot{x}(\mathrm{t}), 0 \leq t \leq T \\
\left(Q_{12} u\right)(t)= & \mu D u(0) \operatorname{Sinh}(t)-\mu \int_{0}^{t} D u(s) \operatorname{Cosh}(t-s) d s+\mu \int_{0}^{t} \operatorname{CDu}(s) \operatorname{Sinh}(t-s) d s+\mu C D u(0) \operatorname{Cosh}(t) \\
+ & \frac{\operatorname{Sinh}(t)}{\operatorname{Sinh}(T)}\left\{\mu C D u(T)-\mu D u(0) \operatorname{Sinh}(T)+\mu \int_{0}^{T} \operatorname{Du}(s) \operatorname{Cosh}(T-s) d s+\mu \int_{0}^{T} \operatorname{CDu}(s) \operatorname{Sinh}(T-s) d s\right. \\
+ & \mu C D u(0) \operatorname{Cosh}(T)\}, 0 \leq t \leq T \\
\left(Q_{22} u\right)(t)= & B u(t)+\mu D^{2} u(t), 0 \leq t \leq T
\end{aligned}
$$

The proof of the above results (3.5) to (3.8) can readily be found in [6].

Since our objective is to solve problems of the form (3.1) and (3.2) introducing the modified gradient techniques to the ECGM, then, there is need to discuss the modified gradient techniques as it applies to an ECGM algorithmin what follows:

\section{ECGM Algorithm} is as follows

The ECGM algorithm for iteratively locating the minimum $x^{*}$ of $f(x)$ in $\mathcal{H}$ as described by [7] and [8]

Step 1: Guess the first element $x_{0}, u_{0} \in \mathcal{H}$ and compute the remaining members of the sequence with the aid of the formulae in the steps 2 through 6.

Step 2: Compute the descent direction $p_{x, 0}=-g_{x, 0}$

$$
p_{u, 0}=-g_{u, 0}
$$

Step 3: Set $x_{i+1}=x_{i}+\alpha_{x, i} p_{x, i}$; where $\alpha_{x, i}=\frac{\left\langle g_{x, i}, g_{x, i}\right\rangle_{\mathcal{H}}}{\left\langle p_{x, i}, Q p_{x, i}\right\rangle_{\mathcal{H}}}$

Set $u_{i+1}=u_{i}+\alpha_{u, i} p_{u, i}$; where $\alpha_{u, i}=\frac{\left\langle g_{u, i}, g_{u, i}\right\rangle_{\mathcal{H}}}{\left\langle p_{u, i}, Q p_{u, i}\right\rangle_{\mathcal{H}}}$

Step 4: Compute $g_{x, i+1}=g_{x, i}+\alpha_{x, i} Q p_{x, i}$

Compute $g_{u, i+1}=g_{u, i}+\alpha_{u, i} Q p_{u, i}$

Step 5: Set $p_{x, i+1}=-g_{x, i+1}+\beta_{x, i} p_{x, i} ; \beta_{x, i}=\frac{\left\langle g_{x, i+1}, g_{x, i+1}\right\rangle_{\mathcal{H}}}{\left\langle g_{x, i}, g_{x, i}\right\rangle_{\mathcal{H}}}$

$$
p_{u, i+1}=-g_{u, i+1}+\beta_{u, i} p_{u, i} ; \beta_{u, i}=\frac{\left\langle g_{u, i+1}, g_{u, i+1}\right\rangle_{\mathcal{H}}}{\left\langle g_{u, i}, g_{u, i}\right\rangle_{\mathcal{H}}}
$$

Step 6: If $g_{, i}=0$ for some $i$, then, terminate the sequence; else set $i=i+1$ and go to step 3 .

In the iterative steps 2 through 6 above, $p_{x, i}$ and $p_{u, i}$ denote the descent directions at ith step of the algorithm, $\alpha_{x, i}$ and $\alpha_{u, i}$, are the step lengths of the descent sequences $\left\{x_{i}\right\}$ and $\left\{u_{i}\right\} . g_{x, i}$ and $g_{u, i}$ denote the gradients of $f$ at $x_{i}$ and $u_{i}$ respectively. Steps 3, 4 and 5 of the algorithm reveal the crucial role of the linear operator $\mathrm{Q}$ in determining the step lengths of the descent sequences and also in generating conjugate directions of search.

\section{Computational Results}

The following problems were evaluated using the ECGM algorithm thus:

Problem (P3)

Find the optimal control which gives an extremum value of the functional

$\mathrm{J}=\int_{0}^{1} u^{2}(t) d t$

Subject to the state differential equation

$\dot{x}_{1}(t)=x_{2}$

$\dot{x}_{2}(t)=u$

$x_{1}(0)=1, x_{1}=0, x_{2}(t)$ and $x_{2}(1)$ is not specified.

\section{Problem (P4)}

What is the optimal trajectory and control for the system

$\dot{x}(t)=x(t)+u(t) ; 0 \leq t \leq 1$,

$x(0)=2$ and $u_{0}(t)=1.0$, that minimizes the performance measure

$\mathrm{J}=\int_{0}^{1}\left\{x^{2}(t)+u^{2}(t)\right\} d t$ 
Problem (5)

$\operatorname{Min}_{(x, u)} \int_{0}^{1}\left\{x^{2}(t)+u^{2}(t)\right\} d t$

Subject to the constraint

$\dot{x}(t)=3 x(t)+2 u(t) ; 0 \leq t \leq 1$,

$x(0)=1$

Conclusion

Computationally, the resulting algorithm from the introduction of the gradient modification to the ECGM were tested on a number of optimal control problems with the penalty parameter, $\mu=10$, the results obtained in each cases were presented in Tables 1 to 3. Our results show improved convergence profile over the classical methods considering the analytical solution of each of the problems under review in this paper.

\section{References}

[1] Aderibigbe, F. M.: "An Extended Conjugate Gradient Algorithm for Control Systems $\quad$ with Delay-I", Advances in Modelling \& Analysis, CAMSE Press, Vol. 36, No. 3, (1993), 51-64.

[2] Aderibigbe, F. M. and Adebayo, K. J.: "A Scaled Extended Conjugate Gradient Method", Jour. of Mathematical Sciences, Vol. 23, No. 1, (2011), 189-196.

[3] George M. Siouris,: An Engineering Approach To Optimal Control And Estimation Theory, John Wiley \& Sons, Inc., (1996)

[4] Hasdorff, L., Gradient Optimization and Nonlinear Control, J. Wiley and Sons, New York, (1976).

[5] Kirk, E. Donald,: Optimal Control Theory, An Introduction, Prentice-Hall, Inc. Englewood Cliffs New Jersey, (2004)

[6] Ibiejugba, M. A. and Onumanyi, P.: "Control Operator and Some of Its Applications", J. Math. Anal. Appl. Vol. 103, No. 1, (1984), 31-47.

[7] Otunta, F. O. and Apanapudor, J. S.,: "The Construction of A Hybrid Operator $\tilde{S}$ ”, Jour. of Inst. of Maths. \& Comp. Sciences, Vol. 21, No. 2, (2008), 61-71.

[8] Emmanuel, N.,: "An Extended Conjugate Gradient Method for Solving Equality Constrained Nonlinear Optimization Problems", Canadian Jour. on Computing in Maths, Natural Sciences, Engineering \& Medicine, Vol. 2, No. 3, (2011), 45-49.

Table 1: Solution of Problem (P3)

\begin{tabular}{|l|l|l|l|}
\hline Iteration & \multicolumn{1}{|c|}{$\boldsymbol{x}_{\mathbf{1}}(\boldsymbol{t})$} & \multicolumn{1}{|c|}{$\boldsymbol{x}_{\mathbf{2}}(\boldsymbol{t})$} & $\mathbf{u}(\mathbf{t})$ \\
\hline 0 & 1.0 & 1.0 & -6.0 \\
\hline 1 & 1.084680787 & 0.200711485 & -5.138692382 \\
\hline 2 & 1.080057317 & -0.283180894 & -4.530819237 \\
\hline 3 & 1.030044608 & -0.707073274 & -3.913879238 \\
\hline 4 & 0.940642661 & -1.070965655 & -3.003538923 \\
\hline 5 & 0.817851477 & -1.374858035 & -2.703289238 \\
\hline 6 & 0.667610545 & -1.618750415 & -2.138952363 \\
\hline 7 & 0.496103942 & -1.802642795 & -1.538292308 \\
\hline 8 & 0.309142496 & -1.926535175 & -0.938923187 \\
\hline 9 & 0.102754359 & -1.890427555 & -0.338923845 \\
\hline
\end{tabular}

Analytical Solution: $x_{1}(t)=0.112794359, x_{2}(t)=-1.990427555$ and $u(t)=-0.3389238$

Table 2: Solution of Problem (P4)

\begin{tabular}{|l|l|l|}
\hline Iteration & \multicolumn{1}{|c|}{$\boldsymbol{x}(\boldsymbol{t})$} & \multicolumn{1}{c|}{$\boldsymbol{u}(\boldsymbol{t})$} \\
\hline 0 & 2.0 & 1.0 \\
\hline 1 & 1.7330480936 & 0.6067807621 \\
\hline 2 & 1.5208769341 & 0.3777519129 \\
\hline 3 & 0.9260250723 & 0.2358764292 \\
\hline 4 & 0.8623790085 & 0.0499097773 \\
\hline 5 & 0.8397009848 & 0.0290384055 \\
\hline 6 & 0.7931014220 & -0.1990770602 \\
\hline 7 & 0.7659701924 & -1.0482730595 \\
\hline
\end{tabular}

Analytical Solution: $x(t)=0.793278181$ and $u(t)=-1.049628059$ 
Table 3: Solution of Problem (P5)

\begin{tabular}{|l|l|l|}
\hline Iteration & \multicolumn{1}{|c|}{$\boldsymbol{x}(\boldsymbol{t})$} & \multicolumn{1}{c|}{$\boldsymbol{u}(\boldsymbol{t})$} \\
\hline 0 & 3.0 & 2.0 \\
\hline 1 & 2.83554338 & 1.742165943 \\
\hline 2 & 1.528200439 & 1.475202917 \\
\hline 3 & 0.223892231 & 1.189145383 \\
\hline 4 & 0.140733082 & 0.487161661 \\
\hline 5 & $0.60505448 \mathrm{e}-1$ & 0.382902064 \\
\hline 6 & $0.38598343 \mathrm{e}-1$ & 0.156341626 \\
\hline 7 & $0.16313756 \mathrm{e}-1$ & 0.117387285 \\
\hline 8 & $0.10416489 \mathrm{e}-1$ & $0.60505448 \mathrm{e}-1$ \\
\hline 9 & $0.11872374 \mathrm{e}-2$ & $0.10416489 \mathrm{e}-1$ \\
\hline 10 & $0.7579272 \mathrm{e}-3$ & $0.44013102 \mathrm{e}-2$ \\
\hline 11 & $0.32026735 \mathrm{e}-3$ & $0.28095923 \mathrm{e}-2$ \\
\hline 12 & $0.86393787 \mathrm{e}-4$ & $0.42685168 \mathrm{e}-2$ \\
\hline 13 & $0.55152705 \mathrm{e}-4$ & $0.19483751 \mathrm{e}-3$ \\
\hline 14 & $0.23305251 \mathrm{e}-4$ & $0.99064718 \mathrm{e}-4$ \\
\hline
\end{tabular}

Analytical Solution: $x(t)=2.28296524 e-5$ and $u(t)=9.92364781 e-5$ 